\title{
Categorical representations of the modular group
}

Cooper, Benjamin

\begin{abstract}
Actions of the modular group on categories are constructed. A hyperelliptic involution is used to convert the braid representations underlying Khovanov homology to representations of the modular group.
\end{abstract}

DOI: https://doi.org/10.1515/forum-2013-0203

Posted at the Zurich Open Repository and Archive, University of Zurich ZORA URL: https://doi.org/10.5167/uzh-171955

Journal Article

Published Version

Originally published at:

Cooper, Benjamin (2016). Categorical representations of the modular group. Forum Mathematicum, 28(1):89-99.

DOI: https://doi.org/10.1515/forum-2013-0203 


\title{
Research Article
}

\section{Benjamin Cooper}

\section{Categorical representations of the modular group}

\begin{abstract}
Actions of the modular group on categories are constructed. A hyperelliptic involution is used to convert the braid representations underlying Khovanov homology to representations of the modular group.
\end{abstract}

Keywords: Knot homology, mapping class groups, braid groups

MSC 2010: 20F36, 18G35

Benjamin Cooper: Universität Zürich, Winterthurerstrasse 190, CH-8057 Zürich, Switzerland, e-mail: benjamin.cooper@math.uzh.ch

Communicated by: Frederick R. Cohen

\section{Introduction}

The study of group actions on categories is a rich topic which arises in many areas of mathematics. Such group actions appear when topological field theories are present. An $n$-dimensional topological field theory $\mathrm{Z}$ associates to each $(n-2)$-manifold $\Sigma$ a category $Z(\Sigma)$ upon which the mapping class group $\Gamma(\Sigma)$. In particular, there are equivalences

$$
F_{g}: Z(\Sigma) \rightarrow Z(\Sigma) \quad \text { for each } g \in \Gamma(\Sigma)
$$

When $n=4$, one expects many examples of mapping class groups $\Gamma(\Sigma)$ acting on categories $Z(\Sigma)$, see $[14,19]$. While there are examples of categorical braid group actions in the literature [15, 20, 24, 27], there are very few examples for surfaces of genus greater than zero; although, see [16].

In this paper we present a means by which the categories underlying the Khovanov homology of knots and links can be used to produce categories which are representations of the modular group $\operatorname{SL}(2, \mathbb{Z})$ (the mapping class group of the torus). A hyperelliptic involution is used to reduce the mapping class group of the torus to the mapping class group of the four punctured sphere. This identification allows us to construct several families of modular group representations from braid group representations.

The theory of modular functors shows that it is possible to construct representations for surfaces of arbitrary genus when given a family of braid group representations and a representation of the modular group satisfying some compatibility conditions $[1,18,26]$. In this sense, the special case of $\operatorname{SL}(2, \mathbb{Z})$ actions is an important component of the most general case.

\section{Organization}

In Section 2, definitions related to knot homology and group actions are recalled. In Section 3, we review information about braid groups and mapping class groups. In Section 4, we construct families of categories $\mathcal{R}_{n, k}$ and $\mathcal{K}_{n}$ which support $\operatorname{SL}(2, \mathbb{Z})$ actions.

\section{Algebraic background}

In this section we collect some algebraic background information. We begin by recalling the differential graded categories $\operatorname{Kom}_{1 / 2}^{*}(n)$ and the existence of certain chain complexes $P_{n, k}$ within $\operatorname{Kom}_{1 / 2}^{*}(n)$. The definition of a group action on a category is given and a construction for reducing the gradings is introduced. 


\subsection{Cobordisms}

Dror Bar-Natan's graphical formulation [2] of the Khovanov categorification [14] consists of a series of categories: $\operatorname{Cob}(n)$ and $\operatorname{Kom}(n)$. The construction in Section 4 will take place within categories related to $\operatorname{Kom}(n)$. Definitions are reviewed below. They are the same as those appearing in $[7,8]$ with the exception that we adopt the Kauffman bracket grading convention from Rozansky [23].

\subsubsection{Categories of cobordisms}

Let $R$ be a field.

Definition 2.1. The category of $\mathfrak{s l}_{2}$-cobordisms with $2 n$ boundary points will be denoted by $\operatorname{Cob}(n)$.

In more detail, there is a pre-additive category $\operatorname{Pre-} \operatorname{Cob}(n)$ whose objects are formally $\mathbb{Z}$-graded diagrams. Each diagram is given by an isotopy class of 1-manifolds embedded in a disk $D^{2}$ relative to $2 n$ boundary points. There is a functor

$$
q: \operatorname{Pre}-\operatorname{Cob}(n) \rightarrow \operatorname{Pre}-\operatorname{Cob}(n)
$$

which increases the grading by one. The morphisms of the category Pre-Cob $(n)$ are given by $R$-linear combinations of isotopy classes of orientable cobordisms embedded in $D^{2} \times I$, see [2].

The internal degree of a cobordism $C: q^{i} A \rightarrow q^{j} B$ is the sum of its topological degree and its $q$-degree:

$$
\operatorname{deg}(C)=\operatorname{deg}_{\chi}(C)+\operatorname{deg}_{q}(C) .
$$

The topological degree

$$
\operatorname{deg}_{\chi}(C)=\chi(C)-n
$$

is given by the Euler characteristic of $C$ and the $q$-degree

$$
\operatorname{deg}_{q}(C)=j-i
$$

is given by the relative difference in $q$-gradings.

We impose the relations implied by the requirement that the circle is isomorphic to the direct sum of two empty sets,

$$
\bigcirc \cong q \varnothing \oplus q^{-1} \varnothing
$$

using maps of internal degree zero (see [3]). In doing so, we obtain the category $\operatorname{Cob}(n)$ as a quotient of the additive closure $\operatorname{Mat}(\operatorname{Pre}-\mathrm{Cob}(n))$ of $\operatorname{Pre}-\operatorname{Cob}(n)$.

There is a composition

$$
\otimes: \operatorname{Cob}(n) \times \operatorname{Cob}(n) \rightarrow \operatorname{Cob}(n) .
$$

It is defined by gluing diagrams and cobordisms. The unit $1_{n}$ with respect to this composition is a diagram consisting of $n$ parallel lines.

\subsubsection{Categories of half-graded chain complexes}

Definition 2.2. If $\mathcal{A}$ is an additive category, then $\operatorname{Kom}_{1 / 2}^{*}(\mathcal{A})$ will denote the differential graded category of $\frac{1}{2}$-graded chain complexes in $\mathcal{A}$.

In more detail, the objects of $\operatorname{Kom}_{1 / 2}^{*}(\mathcal{A})$ are chain complexes $C=\left(C_{i}, d_{i}^{C}\right)_{i \in \frac{1}{2} \mathbb{Z}}$ where $C_{i} \in \mathcal{A}$ and $d_{i}^{C}: C_{i} \rightarrow C_{i+1}$. Each chain complex $C$ is bounded from below: $C_{i}=0$ for $i$ sufficiently small.

Suppose that $C=\left(C_{i}, d_{i}^{C}\right)_{i \in \frac{1}{2} \mathbb{Z}}$ and $D=\left(D_{i}, d_{i}^{D}\right)_{i \in \frac{1}{2} \mathbb{Z}}$ are two chain complexes in $\operatorname{Kom}_{1 / 2}^{*}(\mathcal{A})$. Then a map $g: C \rightarrow D$ of $t$-degree $l$ is a collection

$$
g=\left\{g_{i}: C_{i} \rightarrow D_{i+l}\right\}_{i \in \frac{1}{2} \mathbb{Z}}
$$


of maps in $\mathcal{A}$. The vector space of maps with $t$-degree $l$ will be denoted by $\operatorname{Hom}^{l}(C, D)$. There is a composition

$$
\circ: \operatorname{Hom}^{l}(C, D) \otimes \operatorname{Hom}^{m}(D, E) \rightarrow \operatorname{Hom}^{l+m}(C, E)
$$

and there are identity maps $1_{C}=\left\{1_{C_{i}}: C_{i} \rightarrow C_{i}\right\}$ of degree zero.

There is a differential $d: \operatorname{Hom}^{l}(C, D) \rightarrow \operatorname{Hom}^{l+1}(C, D)$ which is defined on a map $g=\left\{g_{i}\right\}$ of $t$-degree $l$ by the formula

$$
(d g)_{i+1}=g_{i+1} \circ d_{i}^{C}+(-1)^{l+1} d_{i+l}^{D} \circ g_{i} .
$$

If $g \in \operatorname{Hom}^{l}(C, D)$ and $h \in \operatorname{Hom}^{m}(C, D)$ are maps of $t$-degree $l$ and $m$ respectively, then the differential $d$ satisfies the Leibniz rule

$$
d(h \circ g)=h \circ(d g)+(-1)^{l}(d h) \circ g .
$$

If $1_{C}: C \rightarrow C$ is the identity map, then $d\left(1_{C}\right)=0$. Let $\operatorname{Hom}^{*}(C, D)=\prod_{l} \operatorname{Hom}^{l}(C, D)$ be the maps from $C$ to $D$. Then the pairs $\left(\operatorname{Hom}^{*}(C, D), d\right)$ are chain complexes and the category $\operatorname{Kom}_{1 / 2}^{*}(\mathcal{A})$ is a differential graded category [25].

Definition 2.3. The homotopy category $\operatorname{Ho}_{1 / 2}(\mathcal{A})$ of $\operatorname{Kom}_{1 / 2}^{*}(\mathcal{A})$ has the same objects as the category $\operatorname{Kom}_{1 / 2}^{*}(\mathcal{A})$. The maps in $\operatorname{Ho}_{1 / 2}(\mathcal{A})$ are given by the zeroth homology groups of the maps in $\operatorname{Kom}_{1 / 2}^{*}(\mathcal{A})$ :

$$
\operatorname{Hom}_{\mathrm{Ho}_{1 / 2}(\mathcal{A})}(C, D)=\mathrm{H}^{0}\left(\operatorname{Hom}^{*}(C, D)\right),
$$

see [25, Definition 1].

Two objects $C$ and $D$ in $\operatorname{Kom}_{1 / 2}^{*}(\mathcal{A})$ are homotopic, $C \simeq D$, when they are isomorphic in the homotopy category $\operatorname{Ho}_{1 / 2}(\mathcal{A})$. A chain complex $C$ is contractible when $C \simeq 0$.

Definition 2.4. The category of $\frac{1}{2}$-graded chain complexes of cobordisms $\operatorname{Kom}_{1 / 2}^{*}(n)$ is the differential graded category of $\frac{1}{2}$-graded chain complexes in $\operatorname{Cob}(n)$ :

$$
\operatorname{Kom}_{1 / 2}^{*}(n)=\operatorname{Kom}_{1 / 2}^{*}(\operatorname{Cob}(n)) .
$$

\subsubsection{Tangle invariants}

Definition 2.5. The skein relation associated to a crossing is the cone complex

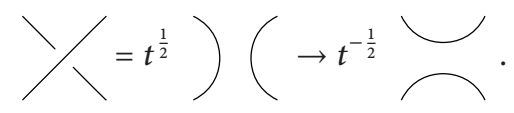

The definition above allows one to associate chain complexes in the category $\operatorname{Kom}_{1 / 2}^{*}(n)$ to diagrams of framed $(n, n)$-tangles. Any two such diagrams which differ by Reidemeister moves 2 or 3 are assigned to homotopy equivalent chain complexes. The first Reidemeister move results in a degree shift, see Property 2 in Section 2.2.1.

\subsection{Projectors}

In [8], V. Krushkal and the author defined a special chain complex $P_{n}$. This construction is summarized by the theorem below. See also [12, 21].

Theorem 2.6. There exists a chain complex $P_{n} \in \operatorname{Kom}_{1 / 2}^{*}(n)$ called the universal projector which satisfies:

(1) $P_{n}$ is positively graded with differential having internal degree zero.

(2) The complex $P_{n} \otimes D$ is contractible for any diagram D which is not the identity $1_{n}$.

(3) The identity object appears only in homological degree zero and only once.

Properties (1)-(3) characterize the projector $P_{n}$ uniquely up to homotopy. 
In [7], M. Hogancamp and the author constructed a series of projectors $P_{n, k} \in \operatorname{Kom}_{1 / 2}^{*}(n)$ where $k=n, n-2$, $n-4, \ldots, n(\bmod 2)$. (The bottom projector $P_{n, n(\bmod 2)}$ is $P_{n, 0}$ when $n$ is even and $P_{n, 1}$ when $n$ is odd.) These higher order projectors are characterized by properties analogous to those mentioned in the theorem above.

Definition 2.7. Suppose that $C=\left(C_{i}, d_{i}^{C}\right)_{i \in \frac{1}{2} \mathbb{Z}}$ is a chain complex in $\operatorname{Kom}_{1 / 2}^{*}(n)$ and let $C_{i}=\bigoplus_{j} C_{i, j}$ so that each object $C_{i, j} \in \operatorname{Pre}-\operatorname{Cob}(n)$ is a diagram. Then the through-degree $\tau(C)$ is the maximum over all of the diagrams $C_{i, j}$ of the minimum number of vertical strands in any cross section of $C_{i, j}$.

In other words, $\tau(C)$ is the largest number of lines connecting the bottom to the top of any diagram $C_{i, j}$ in $C$. Since vertical lines can only be added or removed in pairs, we have

$$
\tau(C) \in\{n, n-2, n-4, \ldots, n(\bmod 2)\} .
$$

Theorem 2.8. For each positive integer $n$, there is a series of higher order projectors $P_{n, k} \in \operatorname{Kom}_{1 / 2}^{*}(n)$ where $k=n, n-2, n-4, \ldots, n(\bmod 2)$ which satisfy the properties:

(1) The $P_{n, k}$ have through-degree $\tau\left(P_{n, k}\right)=k$.

(2) If $D$ is diagram with through-degree $\tau(D)<k$, then

$$
D \otimes P_{n, k} \simeq 0 \text { and } P_{n, k} \otimes D \simeq 0 .
$$

Together with a normalization axiom, analogous to (3) in Theorem 2.6 above, these properties characterize each object $P_{n, k}$ uniquely up to homotopy.

The higher order projectors satisfy orthogonality and idempotence relations. They also fit together to form a convolution chain complex that is homotopy equivalent to the identity object ([7, Section 8]):

$$
P_{n, l} \otimes P_{n, k} \simeq \delta_{l k} P_{n, k} \quad \text { and } \quad 1_{n} \simeq P_{n, n(\bmod 2)} \rightarrow \cdots \rightarrow P_{n, n-4} \rightarrow P_{n, n-2} \rightarrow P_{n, n} .
$$

Remark 2.9. The objects $P_{n}$ and $P_{n, k}$ were originally defined in categories $\operatorname{Kom}(n)$. By construction, every object of $\operatorname{Kom}(n)$ is an object of $\operatorname{Kom}_{1 / 2}^{*}(n)$. This identification allows us to state the theorems as we have above.

\subsubsection{Tangles and twists}

If the projector $P_{n, k}$ is represented by a box, then it satisfies the two properties below.

Property 1 (Drags through tangles). We have

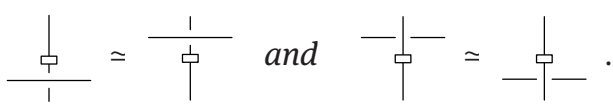

Property 2 (Absorbs twists). We have

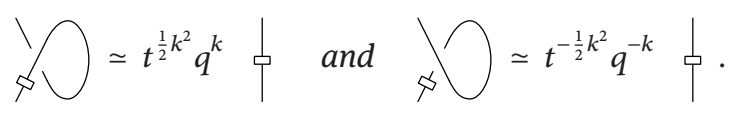

For the degree shifts above to be accurate, the skein relation must be given by equation (2.3). When $n=1$ and $k=1$, Property 2 determines the grading shift associated to the first Reidemeister move.

\subsection{Group actions on categories}

Definition 2.10. If $G$ is a group and $\mathcal{C}$ is a differential graded category, then an action of $G$ on $\mathcal{C}$ is a homomorphism from the group $G$ to the category $\operatorname{End}(\mathcal{C})$ of functors from $\mathcal{C}$ to $\mathcal{C}$ :

$$
F: G \rightarrow \operatorname{End}(\mathcal{C}) \text { such that } F(g h) \simeq F(g) \circ F(h) .
$$

For discussion of dg functors see [25, Section 2.3]. 
If $G=\langle S: R\rangle$ is a presentation for the group $G$, then a group action is obtained by specifying functors $F_{s}, F_{s^{-1}}: \mathcal{C} \rightarrow \mathcal{C}$ for each generator $s \in S$, and homotopy equivalences

$$
F_{s_{1}} \circ F_{s_{2}} \circ \cdots \circ F_{s_{N}} \simeq F_{s_{1}^{\prime}} \circ F_{s_{2}^{\prime}} \circ \cdots \circ F_{s_{M}^{\prime}}
$$

whenever the words $s_{1} s_{2} \cdots s_{N}$ and $s_{1}^{\prime} s_{2}^{\prime} \cdots s_{M}^{\prime}$ coincide by virtue of the relations in $R$. To accomplish this, it suffices to find homotopy equivalences, $F_{s_{1}} \circ \cdots \circ F_{s_{N}} \simeq \operatorname{Id}_{\mathcal{C}}$ for each $r \in R, r=s_{1} \cdots s_{N}$.

When a group $G$ acts on a dg category $\mathcal{C}$, there is an induced action of $G$ on the homotopy category $\mathrm{Ho}(\mathcal{C})$ of $\mathcal{C}$. If $\mathrm{Ho}(\mathcal{C})$ is a triangulated category, then the action of $G$ on $\mathrm{Ho}(\mathcal{C})$ agrees with definitions found in the references $[15,20,24]$.

The notion of group action that is given above is sometimes called weak. A strong action is one in which the homotopy equivalences (2.5) are uniquely determined. We will only prove that the actions defined here are weak, see Remark 4.7.

In Section 4, we will construct $\operatorname{SL}(2, \mathbb{Z})$ actions on categories $\mathcal{R}_{n, k}$ and $\mathcal{K}_{n}$.

\subsection{Reducing the grading}

In this subsection we introduce cyclically graded dg categories $\mathcal{C} / t^{\frac{n}{2}} q^{m}$. If $\mathcal{C}$ is a $\frac{1}{2}$-graded dg category, such as $\operatorname{Kom}_{1 / 2}^{*}(\mathcal{A})$, then there are functors

$$
t^{\frac{1}{2}}, q: \mathcal{C} \rightarrow \mathcal{C}
$$

which increase the $t$ and $q$ gradings by $\frac{1}{2}$ and 1 respectively. The categories $\mathcal{C} / t^{\frac{n}{2}} q^{m}$ are obtained by collapsing the grading so that the identity

$$
t^{\frac{n}{2}} q^{m} \cong \operatorname{Id}_{\mathrm{C} / t^{\frac{n}{2}} q^{m}}
$$

holds in the category End $\left(\mathcal{C} / t^{\frac{n}{2}} q^{m}\right)$. This is accomplished by extending a technique used to define $\mathbb{Z} / 2$-graded dg categories [10, Section 5.1].

Definition 2.11. For each pair of integers, $n, m \in \mathbb{Z}$, there is a $q$-graded differential graded algebra $\left(L_{n, m}, d\right)$ which is given by

$$
L_{n, m}=R\left[t^{\frac{1}{2}}, q\right] /\left(t^{\frac{n}{2}} q^{m}=1\right) \quad \text { and } \quad d=0 .
$$

The ring $R$ is chosen to be the ground field of the category $\mathcal{C}$. The grading of $L_{n, m}$ is determined by the table below:

$$
\begin{array}{cll}
q: & \operatorname{deg}_{q}=1, & \operatorname{deg}_{t}=0, \\
t^{\frac{1}{2}}: & \operatorname{deg}_{q}=0, & \operatorname{deg}_{t}=\frac{1}{2} .
\end{array}
$$

Definition 2.12. Let $\mathcal{C}$ be a $\frac{1}{2}$-graded dg category. An $L_{n, m}$-module in $\mathcal{C}$ is a pair $(C, f)$ consisting of an object $C \in \mathcal{C}$ and a map of graded dg algebras

$$
f: L_{n, m} \rightarrow \operatorname{End}(C) .
$$

The category $\mathcal{C} / t^{\frac{n}{2}} q^{m}$ of $L_{n, m}$-modules in $\mathcal{C}$ is the dg category consisting of $L_{n, m}$-modules and $L_{n, m}$-equivariant maps.

In more detail, the objects of $\mathcal{C} / t^{\frac{n}{2}} q^{m}$ are pairs $(C, f)$ consisting of objects $C \in \mathcal{C}$ and maps $f: L_{n, m} \rightarrow \operatorname{End}(C)$ of differential graded algebras which preserve the gradings. A map $h:(C, f) \rightarrow(D, g)$ of degree $l$ is a map $h: C \rightarrow D$ of degree $l$ in $\mathcal{C}$ which commutes with the action of $L_{n, m}$ on $C$ and $D$ respectively:

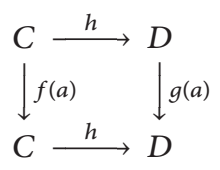

for all $a \in L_{n, m}$. 
The functors $t^{\frac{1}{2}}, q: \mathcal{C} \rightarrow \mathcal{C}$ from equation (2.6) induce functors $t^{\frac{1}{2}}, q: \mathcal{C} / t^{\frac{n}{2}} q^{m} \rightarrow \mathcal{C} / t^{\frac{n}{2}} q^{m}$. For example, if $(C, f)$ is an object of $\mathcal{C} / t^{\frac{n}{2}} q^{m}$, then

$$
t^{\frac{k}{2}} q^{l}(C, f)=\left(t^{\frac{k}{2}} q^{l} C, \varphi \circ f\right)
$$

where $\varphi$ is the natural isomorphism $\operatorname{End}(C) \rightarrow \operatorname{End}\left(t^{\frac{k}{2}} q^{l} C\right)$.

The proposition below shows that the equation $t^{\frac{n}{2}} q^{m} \cong \operatorname{Id}_{\mathcal{C} / t^{\frac{n}{2}}} q^{m}$ holds in $\operatorname{End}\left(\mathcal{C} / t^{\frac{n}{2}} q^{m}\right)$.

Proposition 2.13. Suppose that $t^{\frac{1}{2}}, q: \mathcal{C} / t^{\frac{n}{2}} q^{m} \rightarrow \mathcal{C} / t^{\frac{n}{2}} q^{m}$ denote the functors which increase the $t$-degree and $q$-degree in $\mathrm{C} / t^{\frac{n}{2}} q^{m}$ by $\frac{1}{2}$ and 1 respectively. Then there is an isomorphism

$$
\eta: t^{\frac{n}{2}} q^{m} \cong \operatorname{Id}_{\mathcal{C} / t^{\frac{n}{2}}} q^{m}
$$

in the functor category $\operatorname{End}\left(\mathcal{C} / t^{\frac{n}{2}} q^{m}\right)$.

Proof. The definition of $\mathcal{C} / t^{\frac{n}{2}} q^{m}$ implies that for each object $(C, f) \in \mathcal{C} / t^{\frac{n}{2}} q^{m}$ there are maps $f\left(t^{\frac{1}{2}}\right): C \rightarrow C$ and $f(q): C \rightarrow C$. These maps determine isomorphisms: $\eta_{C}: t^{\frac{n}{2}} q^{m}(C, f) \rightarrow(C, f)$. If $h:(C, f) \rightarrow(D, g)$ is a map in $\mathcal{C} / t^{\frac{n}{2}} q^{m}$, then equation (2.7) implies that $h$ commutes with $\eta_{C}$. So the collection $\eta=\left\{\eta_{C}\right\}$ is a natural transformation $t^{\frac{n}{2}} q^{m} \rightarrow$ Id. The map $\eta$ is the isomorphism in the statement above.

Suppose that $\operatorname{gVect}_{R}$ is the category of graded vector spaces over $R$. Then the example below illustrates the material introduced above.

Example 2.14. An $L_{0, m}$-module in the dg category $\operatorname{Kom}_{1 / 2}^{*}\left(\operatorname{gVect}_{R}\right)$ is a chain complex $C$ on which the grading shift functor $q^{m}: C \rightarrow C$ acts by identity. Similarly, an $L_{n, 0}$-module $C$ in the category $\operatorname{Kom}_{1 / 2}^{*}\left(\operatorname{gVect}_{R}\right)$ is a chain complex on which the functor $t^{\frac{n}{2}}: C \rightarrow C$ acts by identity.

The categories $\operatorname{Kom}_{1 / 2}^{*}\left(\operatorname{gVect}_{R}\right) / t^{0} q^{m}$ and $\operatorname{Kom}_{1 / 2}^{*}\left(\operatorname{gVect}_{R}\right) / t^{\frac{n}{2}} q^{0}$ consist of $q^{m}$-cyclic and $t^{\frac{n}{2}}$-cyclic chain complexes respectively.

There is a forgetful functor

$$
U: \mathcal{C} / t^{\frac{n}{2}} q^{m} \rightarrow \mathcal{C}
$$

which is determined by the assignment $(C, f) \mapsto C$. The functor $U$ has a left adjoint $P: \mathcal{C} \rightarrow \mathcal{C} / t^{\frac{n}{2}} q^{m}$ which sends a chain complex $C$ to $P(C)=L_{n, m} \otimes_{R} C$. If $\pi: \frac{1}{2} \mathbb{Z} \times \mathbb{Z} \rightarrow \frac{1}{2} \mathbb{Z} \times \mathbb{Z} /(n, m)$ is the quotient map, then

$$
P(C)_{i, j}=\bigoplus_{(k, l) \in \pi^{-1}(i, j)} C_{k, l}
$$

where $C_{k, l}$ denotes the value of $C$ in $t$-degree $k$ and $q$-degree $l$. The proposition below records this information.

Proposition 2.15. There is an adjunction

$$
P: \mathcal{C} \mathrm{C} / t^{\frac{n}{2}} q^{m}: U
$$

The proof that the free $L_{n, m}$-module functor $P=L_{n, m} \otimes_{R}$ - is left adjoint to the forgetful functor $U$ is standard, see [17, Section IV.2].

In the remainder of this section we specialize to chain complexes.

Remark 2.16. If $U(C)$ and $U(D)$ are cyclically graded complexes, then $U(C) \otimes U(D)$ is a cyclically graded complex. If $\mathcal{A}$ is a monoidal category, then $\operatorname{Kom}_{1 / 2}^{*}(\mathcal{A}) / t^{\frac{n}{2}} q^{m}$ is a monoidal category.

Proposition 2.17. The homotopy category $\operatorname{Ho}_{1 / 2}(\mathcal{A}) / t^{\frac{n}{2}} q^{m}$ of $\operatorname{Kom}_{1 / 2}^{*}(\mathcal{A}) / t^{\frac{n}{2}} q^{m}$ is a triangulated category.

The proof assumes familiarity with the reference [5].

Proof. The functors $P$ and $U$ commute with totalization.

In more detail, let $\mathcal{C}=\operatorname{Kom}_{1 / 2}^{*}(\mathcal{A}) / t^{\frac{n}{2}} q^{m}$. Given a twisted complex $E=\left\{E_{i}, r_{i j}\right\}$ over $\mathcal{C}([5$, Section 1 , Definition 1]). Let $\alpha(E): \mathcal{C}^{\text {op }} \rightarrow$ Kom be the functor determined by $E$ ([5, Section 1, Definition 3]). By definition,

$$
\alpha(E)(X)=\bigoplus_{i} t^{i} \operatorname{Hom}_{\mathcal{C}}\left(X, E_{i}\right)
$$

The differential of this chain complex is the sum $d+Q$ where $d$ is the Hom-differential from equation (2.2) and $Q=\left(r_{i j}\right)$. 
Let $T(E)=\bigoplus_{i} t^{i} E_{i} \in \mathcal{D}$ be the chain complex with differential $\left(r_{i j}\right)$. Then the chain complex $T(E)$ represents the functor $\alpha(E)$. This is because the functor $h_{T(E)}: \mathrm{C}^{\mathrm{op}} \rightarrow$ Kom associated to $T(E)$ by the Yoneda embedding is given on objects $X$ by

$$
h_{T(E)}(X)=\operatorname{Hom}(X, T(E))=\operatorname{Hom}\left(X, \bigoplus_{i} t^{i} E_{i}\right) \cong \bigoplus_{i} t^{i} \operatorname{Hom}\left(X, E_{i}\right) .
$$

Checking that the differentials agree implies that $h_{T(E)}(X) \cong \alpha(E)(X)$. This isomorphism does not depend on $X$, so $h_{T(E)} \cong \alpha(E)$.

Since each twisted complex $E$ is represented by a chain complex $T(E)$ in $\mathcal{C}$, the category $\mathcal{C}$ is pretriangulated ([5, Section 3, Definition 1]). It follows that the homotopy category $\operatorname{Ho}(\mathcal{C})=\operatorname{Ho}_{1 / 2}(\mathcal{A}) / t^{\frac{n}{2}} q^{m}$ is triangulated ([5, Section 3, Proposition 2]).

The argument above also applies to subcategories.

Corollary 2.18. Cyclic reductions of pre-triangulated subcategories $\mathcal{S} \subset \operatorname{Kom}_{1 / 2}^{*}(\mathcal{A})$ have triangulated homotopy categories $\mathrm{Ho}_{1 / 2}(\mathcal{S}) / t^{\frac{n}{2}} q^{m}$.

The proof of the main theorem shows that the modular group relations hold in a certain category $\varepsilon_{n, k}$ up to a grading shift $\mathfrak{s}_{k}: \varepsilon_{n, k} \rightarrow \varepsilon_{n, k}$. The construction introduced in this section allows us to reduce the grading so that the relations of the modular group hold without the grading shift.

\section{Braid groups and the modular group}

In this section we recall the relationship between the braid group $B_{3}$ and the modular group $\operatorname{PSL}(2, \mathbb{Z})$. Notation established here will be used later.

\subsection{Braid groups}

If $D_{n}$ is the closed disk with $n$ punctures, then the braid group $\mathrm{B}_{n}$ on $n$ strands is given by the path components of the group of boundary and orientation preserving diffeomorphisms of $D_{n}$ :

$$
\mathrm{B}_{n}=\pi_{0}\left(\operatorname{Diff}^{+}\left(D_{n}, \partial D_{n}\right)\right) .
$$

Alternatively, suppose that $\operatorname{Config}_{n}\left(D^{2}\right)$ is the configuration space of $n$ distinct points in the unit disk. Then the symmetric group $S_{n}$ acts on Config ${ }_{n}\left(D^{2}\right)$ by permuting these points and the braid group $\mathrm{B}_{n}$ is the fundamental group of the quotient

$$
\mathrm{B}_{n}=\pi_{1}\left(\operatorname{Config}_{n}\left(D^{2}\right) / S_{n}, x_{0}\right),
$$

see [4, 11]. Each element $[\gamma] \in \mathrm{B}_{n}$ determines a loop $\gamma \in[\gamma], \gamma:(I, \partial I) \rightarrow\left(\operatorname{Config}_{n}\left(D^{2}\right) / S_{n}, x_{0}\right)$. Such a map $\gamma$ factors as $\gamma(t)=\left(\gamma_{1}(t), \ldots, \gamma_{n}(t)\right)$ where $\gamma_{i}: I \rightarrow D^{2}$. The composition of the union $\coprod_{i} \gamma_{i}$ with the fold map is an embedding $\tilde{\gamma}: \bigsqcup_{i} I \hookrightarrow D^{2} \times I$ which determines a braid $\operatorname{im}(\tilde{\gamma})$ in 3 -space. Each such braid can be represented by a diagram consisting of a sequence of crossings $\sigma_{i}$ for $1 \leq i<n$.

When $n=3$, a presentation for the braid group in terms of these generators is given by

$$
\mathrm{B}_{3}=\left\langle\sigma_{1}, \sigma_{2}: \sigma_{1} \sigma_{2} \sigma_{1}=\sigma_{2} \sigma_{1} \sigma_{2}\right\rangle
$$

\subsection{Modular groups}

The modular group $\mathrm{SL}(2, \mathbb{Z})$ is the group of $2 \times 2$ matrices with integer coefficients and determinant 1 :

$$
\operatorname{SL}(2, \mathbb{Z})=\left\{\left(\begin{array}{ll}
a & b \\
c & d
\end{array}\right): a, b, c, d \in \mathbb{Z} \text { and } a d-b c=1\right\} .
$$


It is also the mapping class group of the torus $T$, see [11]. If $I \in \operatorname{SL}(2, \mathbb{Z})$ is the identity matrix, then the center $Z(\operatorname{SL}(2, \mathbb{Z})) \subset \operatorname{SL}(2, \mathbb{Z})$ is the subgroup $\{I,-I\}$. The projective modular group $\operatorname{PSL}(2, \mathbb{Z})$ is the quotient

$$
\operatorname{PSL}(2, \mathbb{Z})=\operatorname{SL}(2, \mathbb{Z}) /\{I,-I\} .
$$

Choosing representatives for the elements $\{I,-I\}$ yields a $\mathbb{Z} / 2$-action on the torus with quotient: $S_{4}^{2}=T /(\mathbb{Z} / 2)$, the four punctured sphere. The group $\operatorname{PSL}(2, \mathbb{Z})$ is the mapping class group of $S_{4}^{2}$. It can be shown that $\operatorname{PSL}(2, \mathbb{Z})$ is isomorphic to the free product $\mathbb{Z} / 2 * \mathbb{Z} / 3$; equivalently,

$$
\operatorname{PSL}(2, \mathbb{Z}) \cong\left\langle a, b: a^{2}=1, b^{3}=1\right\rangle .
$$

\subsection{Relating $B_{3}$ and $\operatorname{PSL}(2, \mathbb{Z})$}

Denote by $q_{n}$ the braid

$$
q_{n}=\sigma_{n-1} \sigma_{n-2} \cdots \sigma_{1} \in \mathrm{B}_{n} .
$$

For instance,

$$
q_{3}=1 /
$$

Multiplying $q_{n}$ by itself $n$ times produces the full twist, $T_{n}=q_{n}^{n}$. The equation

$$
\sigma_{i} T_{n}=T_{n} \sigma_{i} \quad \text { for } 1 \leq i<n
$$

can be seen by dragging $\sigma_{i}$ through from the bottom of $T_{n}$ to the top of $T_{n}$. It follows that $T_{n}$ is contained in the center $Z\left(\mathrm{~B}_{n}\right) \subset \mathrm{B}_{n}$ of the braid group. In fact, $\left\langle T_{n}\right\rangle=\mathcal{Z}\left(\mathrm{B}_{n}\right)$, see [6].

The following proposition tells us that the quotient $B_{3} /\left\langle T_{3}\right\rangle$ is related to the modular group.

Proposition 3.1. The quotient of $\mathrm{B}_{3}$ by the subgroup generated by the full twist $\mathrm{T}_{3}$ is isomorphic to the projective modular group PSL $(2, \mathbb{Z})$,

$$
\mathrm{B}_{3} /\left\langle T_{3}\right\rangle \cong \operatorname{PSL}(2, \mathbb{Z}) .
$$

Proof. If we set $a=\sigma_{1} \sigma_{2} \sigma_{1}$ and $b=\sigma_{1} \sigma_{2}$, then

$$
\mathrm{B}_{3} /\left\langle T_{3}\right\rangle=\left\langle\sigma_{1}, \sigma_{2}: \sigma_{1} \sigma_{2} \sigma_{1}=\sigma_{2} \sigma_{1} \sigma_{2}, T_{3}=1\right\rangle \cong\left\langle a, b: a^{2}=1, b^{3}=1\right\rangle \cong \operatorname{PSL}(2, \mathbb{Z}) .
$$

The groups $\mathrm{B}_{n} /\left\langle T_{n}\right\rangle$ are always related to mapping class groups of punctured spheres, see [4, Section 4.2].

\section{The construction}

In this section we construct sequences of categories $\mathcal{R}_{n, k}$ and prove that each category supports an action of the modular group. We begin by defining categories $\varepsilon_{n, k}$ as extensions of $\operatorname{Kom}_{1 / 2}^{*}(3 n)$ from Section 2.1. The categories $\mathcal{R}_{n, k}$ are obtained from $\varepsilon_{n, k}$ using the construction in Section 2.4. In Theorem 4.5 below, it is shown that there is an action of the group $\operatorname{PSL}(2, \mathbb{Z})$ on each category $\mathcal{R}_{n, k}$. This induces an action of the modular group on $\mathcal{R}_{n, k}$.

The first step is to define a chain complex $E_{n, k}$ consisting of a composition of projectors. This object is illustrated in the proof of Theorem 4.5.

Definition $4.1\left(E_{n, k}\right)$. Suppose that $P_{n, k}$ is the higher order projector from Theorem 2.8. Then set

$$
E_{n, k}=\left(P_{n, k} \sqcup P_{n, k} \sqcup P_{n, k}\right) \otimes P_{3 n, k} .
$$

Definition $4.2\left(\varepsilon_{n, k}\right)$. The category $\varepsilon_{n, k}$ is the full dg subcategory of $\operatorname{Kom}_{1 / 2}^{*}(3 n)$ consisting of objects which are homotopy equivalent to those of the form $C \otimes E_{n, k}$. 
Each object in the category $\varepsilon_{n, k}$ is bigraded. One can shift either the $t$-degree or the $q$-degree of each object using the functors $t^{\frac{1}{2}}$ or $q$. Property 2 in Section 2.2 suggests the following definition.

Definition $4.3\left(\mathfrak{s}_{k}\right)$. The shift functor $\mathfrak{s}_{k}: \varepsilon_{n, k} \rightarrow \varepsilon_{n, k}$ is the composition $\mathfrak{s}_{k}=t^{k^{2}} q^{2 k}$.

If $C \in \mathcal{E}_{n, k}$ is a chain complex, then

$$
\operatorname{deg}_{t}\left(\mathfrak{s}_{k}(C)\right)=\operatorname{deg}_{t}(C)+k^{2} \quad \text { and } \quad \operatorname{deg}_{q}\left(\mathfrak{s}_{k}(C)\right)=\operatorname{deg}_{q}(C)+2 k .
$$

Definition $4.4\left(\mathcal{R}_{n, k}\right)$. The category $\mathcal{R}_{n, k}$ is obtained using the construction from Section 2.4:

$$
\mathcal{R}_{n, k}=\mathcal{E}_{n, k} / \mathfrak{s}_{k} .
$$

Theorem 4.5. The group $\operatorname{PSL}(2, \mathbb{Z})$ acts on the category $\mathcal{R}_{n, k}$.

Proof. Using the presentation $\operatorname{PSL}(2, \mathbb{Z})=\left\langle a, b: a^{2}=1, b^{3}=1\right\rangle$ from Section 3.2, we will construct a functor

$$
\operatorname{PSL}(2, \mathbb{Z}) \rightarrow \operatorname{End}\left(\mathcal{R}_{n, k}\right) \quad \text { where } g \mapsto F_{g} .
$$

Associated to the generators $a$ and $b$ are the chain complexes that are determined by the tangles

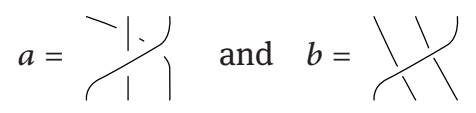

where each line represents an $n$-cabled strand. There are functors, $F_{a}, F_{b}: \mathcal{R}_{n, k} \rightarrow \mathcal{R}_{n, k}$, given by tensoring on the left. To the identity element $1 \in \operatorname{PSL}(2, \mathbb{Z})$, we associate the functor $F_{1}$ where $1=1_{3 n}$ is the diagram consisting of $3 n$ vertical strands. For inverses, $a^{-1}$ and $b^{-1}$, we use the functors $F_{a}$ and $F_{b^{2}}$. To a word $w \cdot w^{\prime} \in\langle a, b\rangle$, we associate the functor $F_{w} \circ F_{w}^{\prime}$. In this manner, we obtain a functor $F_{g}: \mathcal{R}_{n, k} \rightarrow \mathcal{R}_{n, k}$ for each word $g \in\langle a, b\rangle$.

We proceed by checking that the relations $a^{2}=1$ and $b^{3}=1$ hold. Since the braids $a^{2}$ and $b^{3}$ are both full twists with some framing dependency, it suffices to check that this full twist is homotopy equivalent to the identity object in the category $\mathcal{R}_{n, k}$.

Consider what happens when the tangles $a^{2}$ or $b^{3}$ are glued onto $E_{n, k}$. As framed tangles, the full twist $T_{3}$ is isotopic to the tangle pictured on the right below:

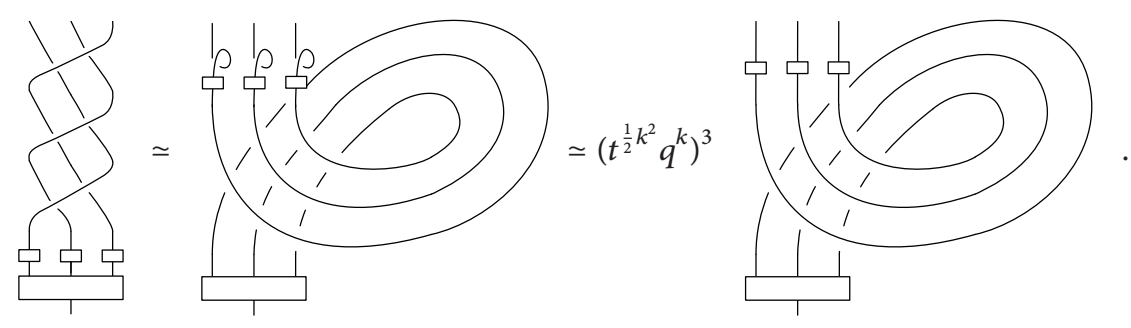

Using the relations of Section 2, we can move the small projectors to the top and absorb the top twists. Using the big projector $P_{3 n, k}$ at the bottom to absorb the middle twist leaves us with

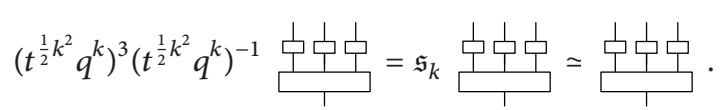

The last equality follows because we have reduced the grading, see Section 2.4. We have shown that the relations $a^{2}=1$ and $b^{3}=1$ hold up to homotopy.

The corollary below notes that the above theorem defines representations of the modular group $\operatorname{SL}(2, \mathbb{Z})$ via pullback.

Corollary 4.6. The group $\mathrm{SL}(2, \mathbb{Z})$ acts on the category $\mathcal{R}_{n, k}$.

Proof. To each element $x \in \mathrm{SL}(2, \mathbb{Z})$ associate the functor $F_{\pi(x)}$ defined in the proof of the theorem where $\pi: \operatorname{SL}(2, \mathbb{Z}) \rightarrow \operatorname{PSL}(2, \mathbb{Z})$. 


\subsection{Concluding remarks}

Remark 4.7. The argument in Theorem 4.5 applies to the subcategories $X_{n}=\operatorname{Kom}_{1 / 2}^{*}(n, n, n, n)$ of $\operatorname{Kom}_{1 / 2}^{*}(4 n)$, see [8, Section 6]. The objects of $X_{n}$ are homotopy equivalent to convolutions of chain complexes of the form



where $D$ is a disjoint union of intervals interfacing between four copies of the projector $P_{n}$. Reducing the grading so that the functor $\mathfrak{s}_{n}$ acts by identity gives a new category $\mathcal{K}_{n}=X_{n} / \mathfrak{s}_{n}$. After fixing one projector, act on the other three by $n$-cabled strands as in the proof above. This is a $\operatorname{PSL}(2, \mathbb{Z})$ representation.

It is possible to argue that the action of $\operatorname{PSL}(2, \mathbb{Z})$ on the category $\mathcal{K}_{n} \otimes \mathbb{F}_{2}$ is strong by using results from previous papers. This material has been omitted.

Remark 4.8. The objects of the category $\mathcal{K}_{n}$ form a basis for the vector space assigned to the four punctured sphere $S_{4}^{2}$ by SU(2) Chern-Simons theory. The quotient map, $T \rightarrow S_{4}^{2}$, described in Section 3.2, identifies the $\mathrm{SU}(2)$ skein space for $S_{4}^{2}$ with the $\mathrm{SO}(3)$ skein space for $T^{2}$ (both with level $r=2(n+1)$ ). See [1, 26].

Remark 4.9. Spin networks of the form (4.1) correspond to $\mathrm{U}_{q} \mathfrak{s l}(2)$-equivariant maps from the $n$th irreducible representation $V_{n}$ to $V_{n}^{\otimes 3}$. The dimension of this space is $n+1$. We may view the categories $\mathcal{K}_{n}$ as a sequence of representations of increasing complexity.

Remark 4.10. It might be interesting to study the groups $K_{0}\left(\mathcal{K}_{n}\right)$ and $K_{0}\left(\mathcal{R}_{n, k}\right)$. The groups $K_{0}\left(\mathcal{E}_{n, k}\right)$ and $K_{0}\left(X_{n}\right)$ are determined in $[7,8]$, but these groups are not the same because the identity $\mathfrak{s}_{k} \cong$ Id implies that there must be other relations.

Remark 4.11. Tensoring the decomposition of identity in equation (2.4) with a chain complex representing the full twist and applying Property 2 shows that the projectors in Theorem 2.8 diagonalize the action of the center $z\left(B_{n}\right)$ on $\operatorname{Kom}_{1 / 2}^{*}(n)$. In order to reduce an arbitrary $G$-action to a $G / Z(G)$-action in a more general setting, one must first find an extension of $\mathcal{C}$ in which the action of the center $\mathcal{Z}(G)$ respects the grading. Some results predict such decompositions for other braid group representations related to knot homologies [13, Theorem 1.1].

\section{References}

[1] B. Bakalov and A. A. Kirillov, Lectures on Tensor Categories and Modular Functors, Univ. Lecture Ser. 21, American Mathematical Society, Providence, 2001.

[2] D. Bar-Natan, Khovanov's homology for tangles and cobordisms, Geom. Topol. 9 (2005), 1443-1499.

[3] D. Bar-Natan, Fast Khovanov homology computations, J. Knot Theory Ramifications 16 (2007), no. 3, 243-255.

[4] J. Birman, Braids, Links and Mapping Class Groups, Ann. of Math. Stud. 82, Princeton University Press, Princeton, 1975.

[5] A. I. Bondal and M. M. Kapranov, Enhanced triangulated categories, Math. USSR Sb. 70 (1991), no. 1, 93-107.

[6] W.-L. Chow, On the algebraic braid group, Ann. of Math. (2) 49 (1948), 654-658.

[7] B. Cooper and M. Hogancamp, An exceptional collection for Khovanov homology, preprint (2012), http://arxiv.org/abs/ 1209.1002.

[8] B. Cooper and V. Krushkal, Categorification of the Jones-Wenzl projectors, Quantum Topol. 3 (2012), no. 2, $139-180$.

[9] B. Cooper and V. Krushkal, Handle slides and localizations of categories, Int. Math. Res. Not. IMRN 2012 (2012), DOI 10.1093/imrn/rns108.

[10] T. Dyckerhoff, Compact generators in categories of matrix factorizations, Duke Math. J. 159 (2011), no. 2, $223-274$.

[11] B. Farb and D. Margalit, A Primer on Mapping Class Groups, Princeton University Press, Princeton, 2011.

[12] I. Frenkel, C. Stroppel and J. Sussan, Categorifying fractional Euler characteristics, Jones-Wenzl projector and $3 j$-symbols with applications to Exts of Harish-Chandra bimodules, Quantum Topol. 3 (2012), no. 2, 181-253.

[13] E. Gorsky and A. Negut, Refined knot invariants and Hilbert schemes, preprint (2012), http://arxiv.org/abs/1304.3328.

[14] M. Khovanov, A functor-valued invariant of tangles, Algebr. Geom. Topol. 2 (2002), 665-741. 
[15] M. Khovanov and P. Seidel, Quivers, Floer cohomology, and braid group actions, J. Amer. Math. Soc. 15 (2002), no. 1, 203-271.

[16] R. Lipshitz, P. Ozváth and D. Thurston, A faithful linear-categorical action of the mapping class group of a surface with boundary, J. Eur. Math. Soc. (JEMS) 15 (2013), no. 4, 1279-1307.

[17] S. MacLane, Categories for the Working Mathematician, Grad. Texts in Math. 5, Springer-Verlag, New York, 1998.

[18] G. Moore and N. Seiberg, Classical and quantum conformal field theory, Comm. Math. Phys. 123 (1989), no. 2, 177-254.

[19] J. Roberts, Rozansky-Witten theory, in: Topology and Geometry of Manifolds (Athens 2001), Proc. Sympos. Pure Math. 71, American Mathematical Society, Providence (2003), 1-17.

[20] R. Rouquier, Categorification of sl(2) and braid groups, in: Trends in Representation Theory and Related Topics, American Mathematical Society, Providence (2006), 137-167.

[21] L. Rozansky, An infinite torus braid yields a categorified Jones-Wenzl projector, preprint (2010), http://arxiv.org/abs/ 1005.3266.

[22] L. Rozansky, A categorification of the stable $S U(2)$ Witten-Reshitikhin-Turaev invariant of links in $\mathbb{S}^{2} \times \mathbb{S}^{1}$, preprint (2012), http://arxiv.org/abs/1011.1958.

[23] L. Rozansky, Khovanov homology of a unicolored $B$-adequate link has a tail, preprint (2012), http://arxiv.org/abs/1203. 5741.

[24] P. Seidel and R. Thomas, Braid group actions on derived categories of coherent sheaves, Duke Math. J. 108 (2001), no. 1, 37-108.

[25] B. Toën, Lectures on dg-categories, in: Topics in Algebraic and Topological K-Theory (Sedano 2007), Lecture Notes in Math. 2008, Springer-Verlag, Berlin (2011), 243-302.

[26] K. Walker, On Witten's 3-manifold invariants, preprint (1991), http://canyon23.net/math/.

[27] B. Webster, Knot invariants and higher representation theory II, preprint (2013), http://arxiv.org/abs/1005.4559.

Received December 15, 2013; revised May 16, 2014. 